\title{
Multi-Modality Imaging of Coronary Artery Fistula with Giant Aneurysms
}

\author{
Hae Won Jung, MD, ${ }^{1}$ Sung Yun Lee, MD, ${ }^{2}$ Yoon Cheol Shin, MD, PhD, ${ }^{3}$ Woo-Ik Chang, MD, PhD, ${ }^{3}$ \\ Jun Hyun Kim, MD, ${ }^{4}$ Hye Ryeong Jo, MD ${ }^{4}$
}

${ }^{1}$ Severance Cardiovascular Hospital, Yonsei University College of Medicine, Seoul, Korea, ${ }^{2}$ Division of cardiology, Department of Internal medicine, Inje University Ilsan Paik Hospital, Goyang, Korea, ${ }^{3}$ Department of Thoracic and Cardiovascular Surgery, Inje University Ilsan Paik Hospital, Goyang, Korea, ${ }^{4}$ Department of Anesthesiology and Pain medicine, Inje University Ilsan Paik Hospital, Goyang, Korea.

\section{ABSTRACT}

A 50-year-old woman was referred to our hospital with a chief complaint of chest pain. Coronary angiography revealed a fistula between the left anterior descending artery and pulmonary artery with giant aneurysms. Although coronary angiography is considered the standard tool to confirm a coronary artery fistula, the patient in this case underwent successful surgical repair with the aid of multi-modality imaging.

\section{INTRODUCTION}

Coronary artery fistulas (CAFs) are rare, with an incidence of only $0.1 \%$ in patients undergoing coronary angiography (CAG) (Vavuranakis 1995). CAFs are typically diagnosed by coronary angiography. However, limited case reports have described the use of multi-modality imaging for the evaluation of CAFs. Herein, we describe the case of a woman with CAF with giant aneurysms, which were successfully treated using multi-modality imaging.

\section{CASE REPORT}

A 50-year-old woman with hypertension was referred to our hospital for evaluation of exertional chest pain. Her vital signs were stable upon examination. A continuous murmur was heard at the left mid sternal border. Transthoracic echocardiogram (TTE) revealed preserved left ventricular systolic function with left atrial enlargement, and no abnormal color flow signal was noted. Subsequent coronary angiography demonstrated a CAF with giant aneurysms in the proximal left anterior descending artery (LAD) which appeared to drain into the main pulmonary artery (PA) (Figure 1, A and B). Because of the presence of the giant aneurysms, we could not evaluate the proximal LAD and determine the apparent relation of the CAF and other structures. To evaluate the proximal $\mathrm{LAD}$ and localize the CAF, we decided to perform multidetector computed tomography

Received Fanuary 23, 2017; received in revised form May 9, 2017; accepted fune 13, 2017.

Jun Hyun Kim, MD, Department of Anesthesiology and Pain medicine, Inje university Ilsan Paik Hospital, 2240 Daebwa-dong, Ilsanseo-gu, Goyang-si, Gyeonggi-do, 10380 Korea; Tel: +82-31-910-7067, Fax: +82-31-910-71845 (e-mail:ishmae@paik.ac.kr).

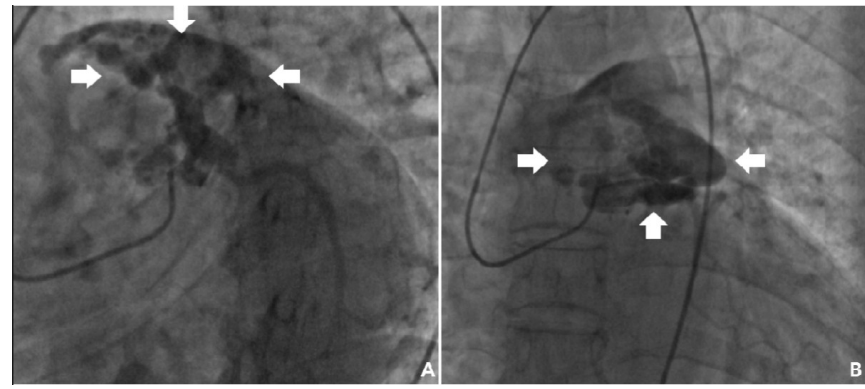

Figure 1. Coronary angiography demonstrating coronary artery fistula with giant aneurysms (white arrow head) between the left anterior descending artery

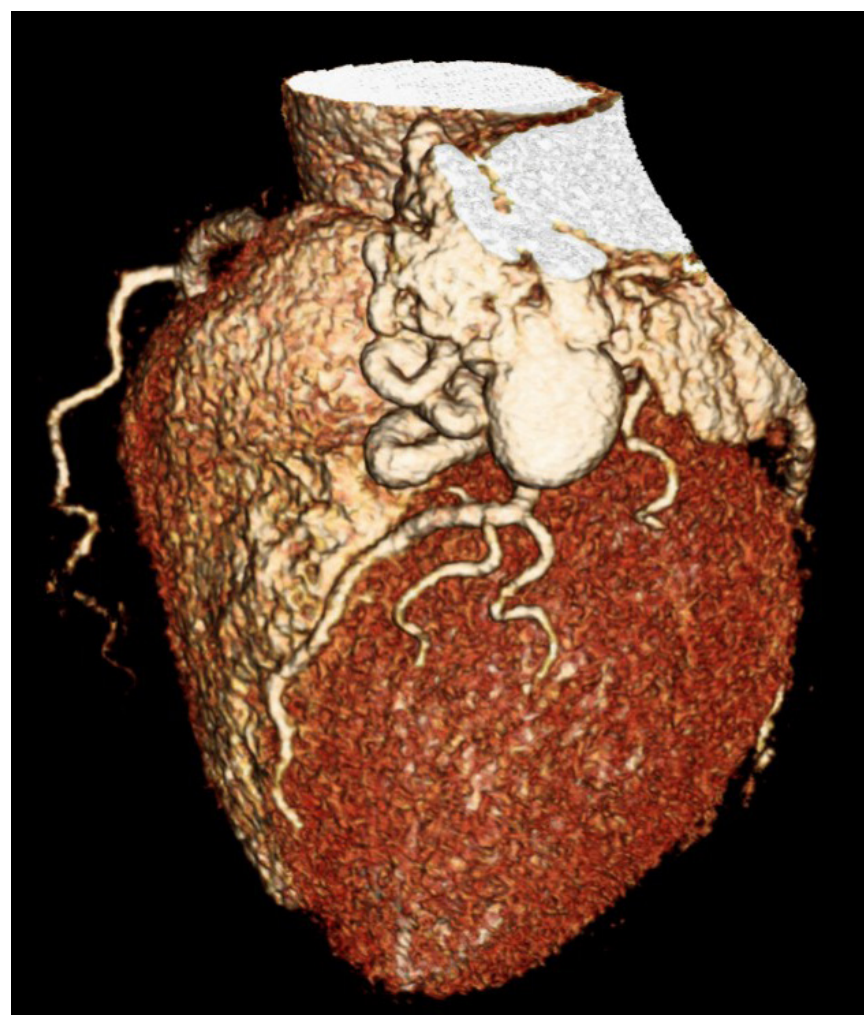

Figure 2. The volume-rendered image from multidetector computed tomography angiography demonstrating a coronary-pulmonary artery fistula with giant aneurysms. 

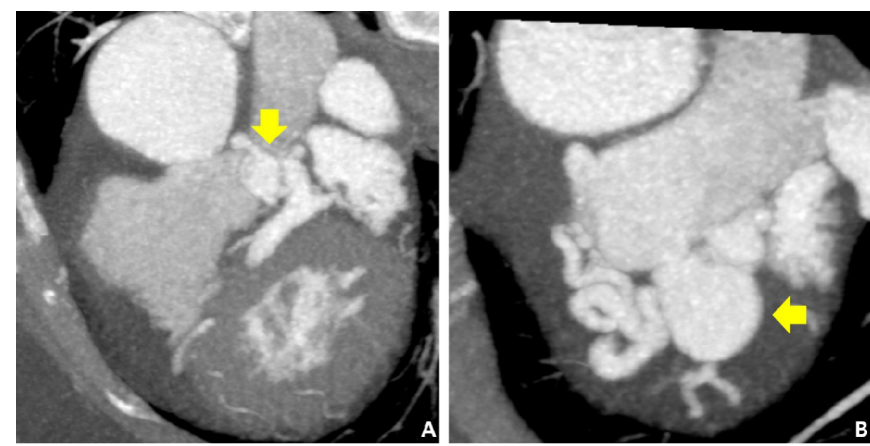

Figure 3. Multidetector computed tomography angiography revealed coronary artery fistula from proximal left anterior descending artery to pulmonary trunk with two aneurysms at adjacent left anterior descending artery ostium (yellow arrowhead) (A) and anterior portion of pulmonary trunk (yellow arrowhead) (B).
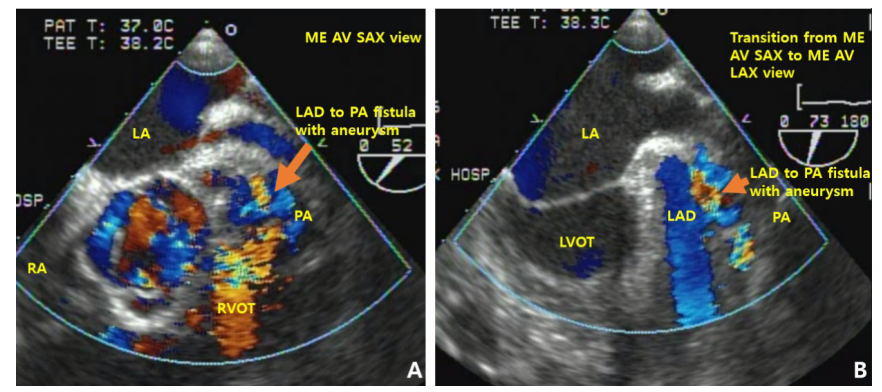

Figure 4. The LAD artery to PA fistula was visualized on the ME AV SAX view $(A)$ and fully visualized by tracing the vessel during transition from the ME AV SAX view to the ME AV LAX view (B). ME AV SAX view indicates midesophageal aortic valve short-axis view; ME AV LAX, midesophageal aortic valve long-axis view; LAD, left anterior descending; PA, pulmonary artery; LA, left atrium; RA, right atrium; RVOT, right ventricular outflow tract.

(MDCT) angiography using a dual-source 320-slice MDCT scanner (Figure 2). MDCT angiography revealed a CAF from the proximal LAD to the pulmonary trunk, with two aneurysms (Figure 3, A and B). No significant plaque or stenosis of the proximal $\mathrm{LAD}$ was noted. Surgical ligation of the CAF was scheduled with consideration for the potential risk of rupture of the aneurysms. The CAF was identified using TEE, and blood flow was recorded prior to surgical separation of the fistula. As shown in Figure 4, the fistula between the LAD and PA was visualized on the midesophageal aortic valve short-axis (ME AV SAX) view (Figure 4, A), and fully visualized by tracing the vessel during transition from the ME AV SAX view to the midesophageal aortic valve long axis (ME AV LAX) view (Figure 4, B). The operation was performed through a midline sternotomy approach using cardiopulmonary bypass with bicaval cannulation. Two aneurysms and the surrounding $\mathrm{CAF}$ were found left of the PA trunk (Figure 5, A and B). After separating the fistula from the adjacent tissue, the fistula was partially ligated. Blood flow in the fistula was decreased as observed by TEE color Doppler monitoring. Over the following 10 minutes, no heart wall motion abnormalities were detected, as determined by TEE. The fistula was completely ligated and the aneurysms
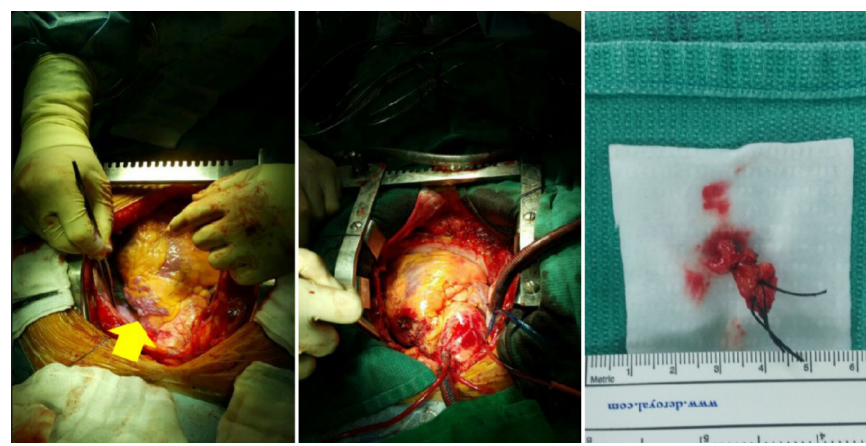

Figure 5. Two aneurysms and the surrounding coronary artery fistula were found left of the pulmonary artery trunk (yellow arrowhead) (A) The fistula was completely ligated and aneurysms were excised $(B, C)$.

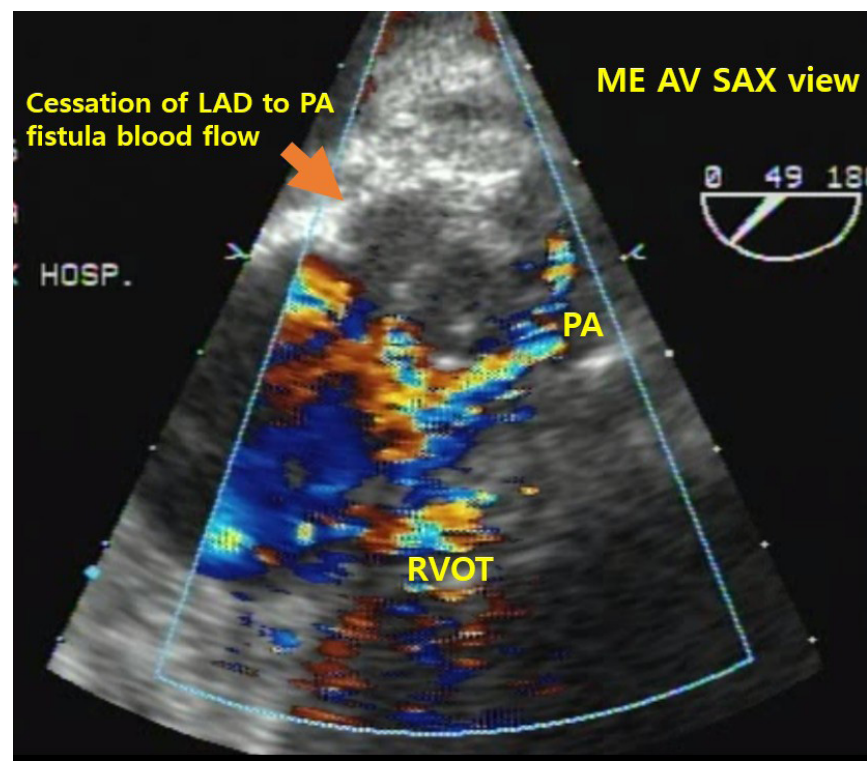

Figure 6. After complete ligation of fistula, cessation of fistula blood flow was confirmed by transesophageal echocardiography. ME AV SAX view indicates midesophageal aortic valve short-axis view; LAD, left anterior descending; PA, pulmonary artery; RVOT, right ventricular outflow tract.

were excised (Figure 5, C). Cessation of fistula blood flow was confirmed by TEE (Figure 6). Seven days after surgery, we performed follow-up imaging of the lesion with MDCT angiography. MDCT angiography demonstrated complete removal of previous engorged fistulous coronary arteries (Figure 7, Figure $8, \mathrm{~A}$ and $\mathrm{B})$. The patient was discharged without complications and remains asymptomatic at this time.

\section{DISCUSSION}

CAFs are anomalous connections of the coronary arteries. The majorities of CAFs are asymptomatic and follow a benign course. It is commonly believed that the CAF lesion is incidentally detected on coronary angiography. Generally, symptoms develop depending on the amount of the 


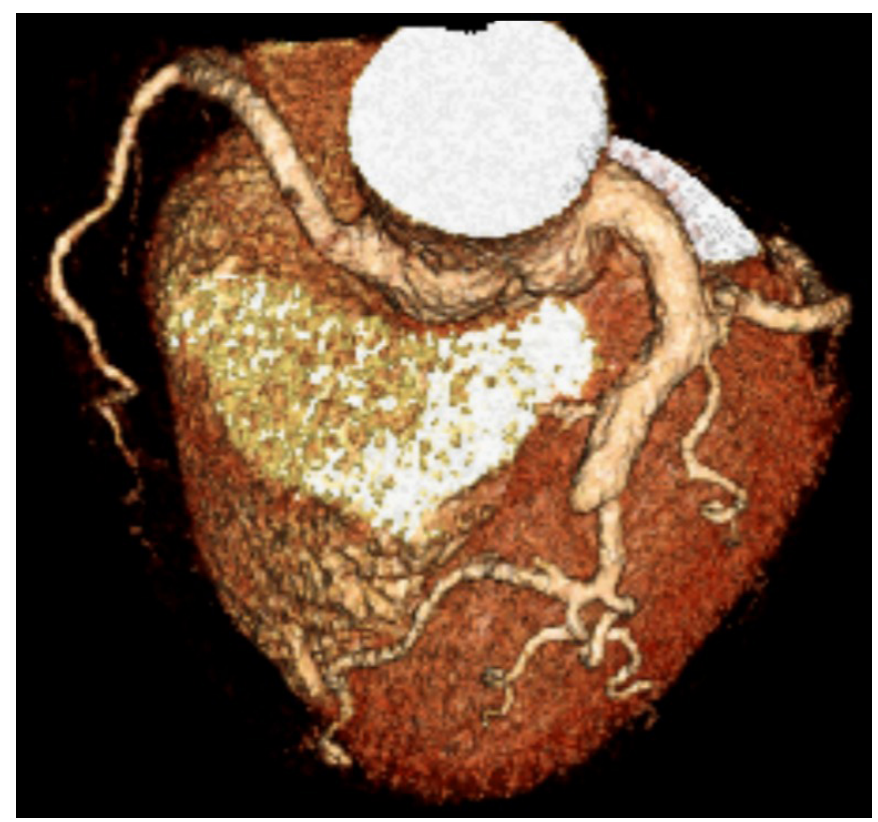

Figure 7. The volume rendered image from multidetector computed tomography angiography demonstrated complete removal of previous multiple engorged fistulous coronary arteries.

left-to-right shunt or the presence of coronary steal phenomenon caused by the fistulas; symptoms in adults usually include, angina, exertional dyspnea, syncope, or palpitations [Challoumas 2014].

Coronary angiography has traditionally been used to diagnose CAFs; however, this invasive procedure is not optimal as it carries inherent risks, including a $0.1 \%$ risk of mortality and vascular complications. Furthermore, it can be difficult to delineate the precise course of CAF anatomy, particularly the drainage sites, which can be diluted by the contrast medium, and the images may be ambiguous cases of anomalous and tortuous blood vessels [Ali 2016]. Some studies have demonstrated the utility of MDCT angiography in assisting surgeons with preoperative planning, as well as follow-up studies. The multiplanar reformation can clearly demonstrate the sites of origin and termination of abnormal blood vessels, and the volume-rendered images can provide an overview of the heart as well as its vascular anatomy, enabling surgeons to better understand the anatomical complexity before surgery [Zhou 2015].

The optimal management strategy for asymptomatic fistulas remains controversial. However, there is general agreement that patients with symptomatic CAF should receive treatment. According to the American College of Cardiology/American Heart Association (ACC/AHA) guidelines, percutaneous or surgical closure is a Class I recommendation for large fistulas regardless of symptoms, and for small to moderate size fistulas with evidence of myocardial ischemia, arrhythmia, ventricular dysfunction, ventricular enlargement, or endarteritis [Warnes 2008]. For CAF with aneurysm, surgical ligation or transcatheter intervention may be indicated to prevent spontaneous rupture, dissection,
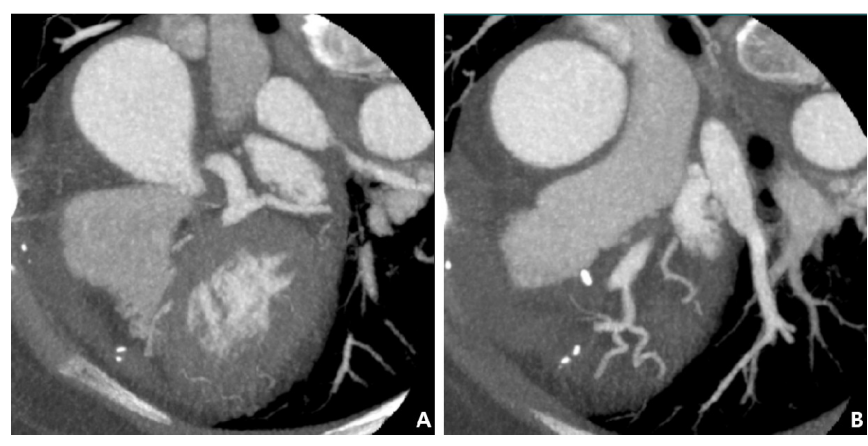

Figure 8. Multidetector computed tomography angiography demonstrated complete removal of previous multiple engorged fistulous coronary arteries (A, B).

myocardial ischemia, or thromboembolic events. Indications for surgery include large CAFs with high flow shunts, multiple communications and terminations, aneurysmal formation, and the need for simultaneous coronary bypass [Piao 2015]. Because the patient in this case had multiple communications and giant aneurysms, we selected surgery as a therapeutic method.

Said et al have emphasized that attention should be paid to the possibility of perioperative myocardial infarction, as this appeared to be a significant factor determining survival rates. In their study, out of the 46 patients who underwent surgical CAF repair, 5 (11\%) patients developed postoperative myocardial infarction. These results demonstrate the importance and relatively high incidence of this serious complication as well as the necessity of intraoperative TEE [Said 2013].

Although there are no guidelines for post-treatment follow-up of adult patients with congenital CAF, some physicians recommended MDCT angiography as a follow-up method based on the clinical features [Saboo 2014]. Comparison of pretreatment MDCT images with post-fistula closure follow-up studies is helpful for evaluating the risk of fistula recurrence leading to recanalization and dilation of the coronary arteries, as well as for assessing the degree of thrombosis following the intervention.

This case demonstrates the value of using multi-modality imaging in the diagnosis and surgical treatment of CAF. We were able to diagnose CAF with giant aneurysms using MDCT angiography, and employed intraoperative TEE to ensure a safe and successful surgical CAF ligation. We were further able to confirm the successful results of surgical resection with follow-up MDCT angiography.

\section{REFERENCES}

Ali A, Colledge J, Sri I, Missouris C. 2016. CT: the imaging of choice in the diagnosis of coronary artery fistulae. BJR Case Rep 2:20150492.

Challoumas D, Pericleous A, Dimitrakaki IA, Danelatos C, Dimitrakakis G. 2014. Coronary arteriovenous fistulae: A review. Int J Angiol 23:1-10.

Piao ZH, Jeong MH, Jeong HC, et al. 2015. Coronary artery fistula with giant aneurysm and coronary stenosis treated by transcatheter embolization and stent. Korean Circ J 45:245-7. 
Saboo SS, Juan YH, Khandelwal A, et al. 2014. MDCT of congenital coronary artery fistulas. AJR Am J Roentgenol 203:W244-52.

Said SM, Burkhart HM, Schaff HV, et al. 2013. Late outcome of repair of congenital coronary artery fistulas--a word of caution. J Thorac Cardiovasc Surg 145:455-60.

Vavuranakis M, Bush CA, Boudoulas H. 1995. Coronary artery fistulas in adults: Incidence, angiographic characteristics, natural history. Cathet Cardiovasc Diagn 35:116-20.
Warnes CA, Williams RG, Bashore TM, et al. 2008. ACC/AHA 2008 Guidelines for the Management of Adults with Congenital Heart Disease: Executive Summary: a report of the American College of Cardiology/American Heart Association Task Force on Practice Guidelines (writing committee to develop guidelines for the management of adults with congenital heart disease).

Circulation 118:2395-2451. Zhou K, Kong L, Wang Y, et al. 2015. Coronary artery fistula in adults: Evaluation with dual-source CT coronary angiography. Br J Radiol 88:20140754. 